COMENTARIO

\title{
El camino de la biotecnología en la Universidad Nacional Agraria La Molina, Perú
}

\author{
The path of Biotechnology at the Universidad Nacional Agraria La \\ Molina, Peru
}

Laboratorio de Micología y Biotecnología, Universidad Nacional Agraria La Molina, Av. La Molina s/n Lima 12, Perú. Email: mgclmb@lamolina.edu.pe

\section{Marcel Gutiérrez-Correa}

\begin{abstract}
Resumen
Se realiza un relato histórico sobre los cincuenta años que tiene la carrera de biología en la Universidad Nacional Agraria La Molina y la gestación, inicio, desarrollo y maduración de la biotecnología en esta universidad. Se describen algunos pormenores de las tres grandes estructuraciones curriculares que finalmente derivan al establecimiento del área de biotecnología en el pregrado y en el doctorado así como a los logros alcanzados. El desarrollo de la biotecnología en la UNALM ha influenciado el desarrollo de esta área en el Perú no solo en las adaptaciones curriculares que se han producido en varias universidades del país sino también en las investigaciones biotecnológicas que se llevan a cabo en universidades e institutos de investigación en las diferentes regiones del país. En este devenir histórico se concluye que el balance es muy positivo y que la mejor corriente epistemológica es la que nos lleva mediante la investigación a encontrar verdades usables en la solución de los problemas del país y, también por qué no, del planeta.

Palabras clave: historia de la biología en el Perú; biotecnología; universidad; curriculum.

\section{Abstract}

In this paper, I make a historical account of the fifty years that have the degree in biology at the Universidad Nacional Agraria La Molina and gestation, and the beginning, development and maturation of biotechnology in this university. We describe some details of the three most important curricular modifications that led to the establishment of the biotechnology area in the curricula of undergraduate and doctoral programs, as well as their main achievements. The development of biotechnology at the UNALM has influenced the development of this area in Peru not only in the curricular changes that have occurred in several Peruvian universities, but also in the biotechnology research being carried out in many regions of the country. In this historical process, I conclude that the balance is positive and that the best epistemology stream is that which leads through research to find truths usable for solving the problems of Peru and also why not the planet.
\end{abstract}

Keywords: history of biology in Peru; biotechnology; university; curriculum.
Amicus Plato sed magis amica veritas - Aristóteles

Caminante, no hay camino, se hace camino al andar-Antonio Machado

\section{Introducción}

Han transcurrido cincuenta años desde que en 1962 se creó la Carrera de Biología en la Facultad de Ciencias de la Universidad Nacional Agraria La Molina (UNALM). Mi relación con la carrera lleva 44 años, primero como estudiante desde 1968 y luego como profesor desde 1975 (37 años) por lo que me ha tocado ser parte de su desarrollo histórico, incluyendo el periodo 1988 a 1992 como presidente de la Comisión de Currículo de la Facultad de Ciencias encargada de la última gran reestructuración curricular. Es indudable que relatar la historia es una tarea extremadamente difícil por el inevitable sesgo del relator, aunque el respetar los hechos y las evidencias que como científicos estamos acostumbrados espero logre minimizarlo. Sin embargo, a pesar que los tiempos y hechos que se relatan tratan de ser los más ajustados a la verdad, es inevitable analizarlos y en ello, muy a mi pesar, la subjetividad puede inmiscuirse por lo que pido disculpas si institucionalmente o personalmente puedan sentirse afectados y porque, desafortunadamente, he de formar parte del relato. Adicionalmente, un relato histórico detallado de los primeros 25 años ha sido realizado por el Dr. Pedro Aguilar Fernández (Aguilar 1986).
Solo 10 años antes de la creación de la carrera, Hersey y Chase (1952) habían hecho la comprobación definitiva que el ADN era la base del material hereditario y genético de los seres vivientes y un año después Watson, Crick, Franklin y Wilkins (Franklin \& Gosling 1953a, b, Watson \& Crick 1953a, b, Wilkins et al. 1953) proponían la estructura del ADN. En los dos años previos a la creación de la carrera, ya se había dilucidado el código genético y Jacob y Monod (1961a, b) proponían el modelo del operón para explicar la regulación de la expresión génica. Mientras tanto, la biología celular descriptiva había avanzado sustancialmente. La botánica y la zoología eran las áreas que más habían avanzado en el país y la genética era particularmente avanzada en la UNALM. Dentro de ese contexto y considerando que existía una diversidad profesional entre los profesores de biología, se inicia la carrera de biología para formar biólogos mediante un "curriculum fundador" que funcionó hasta inicios de los años 70 dentro de un esquema de facultad. No había orientaciones y los cursos de biología estaban relacionados a la biología descriptiva pero había muchos cursos de otros departamentos y facultades (los nuevos enfoques moleculares tardarían más tiempo en ser incorporados en el plan curricular). 


\section{De 1962 a 1972: El currículo fundador}

Existió una fuerte y amplia comunicación entre los estudiantes y los profesores no solo del departamento de biología sino también de otros departamentos. Hay que reconocer, y agradecer, que a pesar de la diversidad profesional de los profesores (agrónomos, biólogos, médicos veterinarios, zootecnistas y químico-farmacéuticos) y el enfoque que impartían de utilidad de la biología para solucionar problemas del país, la enseñanza fue profunda y amplia a la vez pero también abierta a desarrollarse. Se nos acostumbró al uso del libro texto y a la lectura de textos complementarios y aún de artículos científicos pues, "la formación profesional va más allá de los cursos curriculares”. Es así que los estudiantes de mi generación solicitaron nuevos cursos y los profesores nos secundaron en ello. Cursos como Bioquímica II (metabolismo), Fisiología Microbiana, Genética Microbiana, Virología, Microorganismos Zoopatógenos, Histología y Embriología Animal y otros, fueron abiertos e incorporados a nuestro plan curricular.

El curso de Fisiología Celular, luego el curso de Biología II, fue la consecuencia de un hermoso curso teórico-práctico que ofreció la Dra. Emma Loza a su regreso del postgrado, conjuntamente con su profesor asesor el Dr. Plaut y que se denominó “Tópicos Especiales en Citología” donde se nos enseñó en detalle las principales técnicas microscópicas, sus fundamentos y el análisis de imágenes, conocimientos que han perdurado en el tiempo. Estos conocimientos los he trasmitido a mis estudiantes y me han permitido, junto con la Dra. Gretty K. Villena, haber publicado importantes artículos de nuestros estudios de biopelículas mediante microscopía electrónica de barrido y microscopía laser confocal de barrido (Villena \& Gutiérrez-Correa 2007, Villena et al. 2010; 44 citaciones en 3 ańos). Otro gran curso creado a nuestro pedido fue el de Enzimología ofrecido por el Dr. Jacques Schonberg, un sabio, el curso más difícil que he tenido en toda mi vida pero con un impacto fundamental en mi investigación.

\section{De 1973 a 1992: El segundo currículo}

Hay que resaltar que el periodo comprendido entre 1968 a 1973 fue muy convulsionado, en lo político con una dictadura militar, y su influencia social (reforma agraria y socialismo), y en lo institucional con un cisma en el gobierno de la UNALM, recesos y el cambio de la Ley Universitaria que eliminaba las facultades y establecía los Programas Académicos y el departamentalismo (curiosamente una estructura norteamericana promovida por un gobierno de corte socialista), y en lo telúrico con el terremoto de 1970 (y el siguiente en 1974). En el mundo, la guerra fría, la guerra de Vietnam y el movimiento Hippie también jugaron un papel que habrá que analizar posteriormente. Sin embargo, la nueva Ley Universitaria trajo consigo la primera gran reestructuración curricular con la que se consolidó el segundo currículo integrado de biología que incorporó los nuevos cursos, con cuatro orientaciones y que empezó a funcionar oficialmente en 1973; tuvo un enfoque de biología dinámica y funcional. En mi opinión, la Ley de la dictadura fue muy nociva para el sistema universitario, entre varios aspectos, por el predominio del departamentalismo y el aislacionismo intelectual a que ha conllevado y por la reestructuración presupuestal. Antes de 1973 las universidades manejaban más autónomamente su presupuesto, además diferenciado, y financiaban su propia investigación. Un gran error cometido por el Programa Académico de Ciencias fue la cancelación de la Carrera de Química. La nueva y aún vigente Ley Universitaria 23733 se promulgó a finales de 1983 con la cual se restituyó el sistema de facultades.

En febrero de 1983, como consecuencia de un gran curso para profesores de secundaria que me tocó coordinar, se elaboró un texto de biología que aún se encuentra en la Biblioteca Agrícola Nacional con el código QH308.2.G8 (Gutiérrez-Correa 1983), que luego fue usado también para el curso de Biología General por algunos profesores. En la parte teórica con 31 unidades participaron: Pedro Aguilar (3 unid,) Luis Basto, qepd, (6 unid.), Emma Loza (5 unid.), M. Gutiérrez-Correa (11 unid.), Inés Redolfi ( 6 unid.); en la parte práctica con 10 sesiones participaron, entonces aún Jefes de Práctica, Eduardo Gómez-Cornejo (2 ses.), Antonietta Gutiérrez (4 ses.) y Doris Zúniga (4 ses.). En el contexto de este relato es pertinente transcribir el primer párrafo del prólogo:

"Actualmente, la biología es, y con mucha razón, la más emocionante de las ciencias. Los grandes avances ocurridos en la biología en los últimos diez años han sobrepasado la barrera de lo revolucionario. Hasta hace algunos años parecía imposible que organismos tan diferentes, en términos evolutivos, como el hombre y las bacterias pudiesen llegar a tener mecanismos moleculares muy similares a tal grado que hoy día es común la producción bacteriana de insulina. Esto ha sido alcanzado con el conocimiento de los mecanismos de replicación del DNA, con la concepción molecular del gen y con la premisa evolutiva de provenir de un antepasado común. Esto permite que puedan manipularse los genes en forma tal que dentro de los próximos años no habrá macromolécula humana que no pueda ser producida por las bacterias. Estos aspectos son estudiados y desarrollados por la Ingeniería Genética”.

Ese libro también contribuyó a la adopción final en el Departamento de Biología del sistema de cinco reinos que hasta entonces había tenido mucha resistencia, aún por algunos docentes muy jóvenes, a pesar que se aceptaba en el mundo desde 1969 (Whittaker 1969) y ya estaba siendo reemplazado por el sistema de seis reinos de Woese et al. (1977) y más tarde por el sistema de tres dominios de Woese et al. (1990) y nuevas visiones (Zakaib 2011).

\section{Los inicios de la biotecnología}

Durante ese periodo de convulsión, en el mundo la biología avanzaba a pasos agigantados y ya en 1973 surge la ingeniería genética y el advenimiento de la biotecnología moderna en la que la biología molecular se vincula muy fuertemente con la ingeniería biológica posibilitando la generación de nuevas tecnologías para la producción de bienes y servicios. El término "biotecnología" había sido acuñado en 1917 por el húngaro Karl Ereky (Fári \& Kralovánszky 2006).

En 1980, llega a mis manos el Informe que tres grandes científicos franceses presentan, a su pedido, al Presidente de la República de Francia en el que analizan el estado de desarrollo de las ciencias biológicas y la biotecnología y sus proyecciones futuras, recomendando tomar medidas urgentes para que Francia no quede rezagada:

[“...Développer la microbiologie et le genie génétique, former des chercheurs et des ingénieurs, intensifier l'activité des centres compétentes, tout cela constitue une priorité absolue our les mois 
à venir. En dépendent les résponses qu'il sera possible d'apporter à de nombreux problèmes, en particulier l'alimentation des hommes.'] (Gros et al. 1979).

Este libro fue muy ilustrativo y de gran impacto en los años venideros.

Aunque inicialmente las biotecnologías eran desarrolladas con bacterias, en 1984 científicos de la Universidad de Gante demuestran la aplicabilidad de la ingeniería genética a las plantas (Herrera-Estrella 1983, De Block et al. 1984). En ese año, el CONCYTEC nos encarga a un grupo de profesores universitarios (UNALM, UPCH, UNMSM, UNT) organizar un seminario para analizar esta nueva disciplina que causaba furor en el mundo. En el país, como es usual, estábamos rezagados respecto a otros países latinoamericanos. En la UNALM, algo habíamos aprendido sobre el tema y también para competir por fondos (cosa que no era popular en la época) al aliarnos con la empresa Sociedad Paramonga Ltda. y ganar un proyecto financiado por el ITINTEC. También se iniciaban trabajos en micropropagación de plantas tanto en biología como en agronomía. En agosto de 1985, vino a nuestro laboratorio como profesor visitante el Dr. Robert P. Tengerdy de Colorado State University que compartió conmigo el curso de Fisiología Microbiana y se organizó el primer curso a nivel nacional de biotecnología al que concurrieron más de 400 personas de todo el país. Como producto de este curso, se elaboró un libro que se encuentra en la Biblioteca Agrícola Nacional con el código TP248.2.G8, con la participación de varios profesores de biología, química, agronomía y también de otras universidades (Gutiérrez-Correa 1985).

En la segunda mitad de 1986, el CONCYTEC crea la Primera Comisión Nacional de Biotecnología conformada por 25 personas procedentes de la UPCH, UNMSM, Sinquisa y solo un representante de la UNALM el cual, sorpresivamente, fue elegido como presidente. En poco menos de un ańo, después de bastante trabajo, presentamos a la Presidencia del CONCYTEC el primer Plan Nacional de Biotecnología que incluía en un periodo de 5 años la formación de recursos humanos, el establecimiento de centros de excelencia en la UNALM (agroindustria), UPCH (biología molecular y salud) y UNMSM (plantas y farmoquímica), equipamiento moderno y el financiamiento de investigaciones prioritarias y de 2 a 3 proyectos pilotos; el presupuesto anual del Plan correspondía al 8,8\% del presupuesto del CONCYTEC (en ese momento uno de los más abultados presupuestos de Latinoamérica). Desafortunadamente, la propuesta fue considerada contraria a la política populista del "caos premeditado" conducida por el CONCYTEC y, por tanto, no fue aprobada y el país perdió una gran oportunidad que no ha vuelto a tener.

A partir de 1985 hasta mediados de 1992 se desarrolló en la UNALM el Proyecto de Asistencia al Desarrollo Institucional (PADI)/UNALM/USAID/Ministerio de Agricultura con el cual se financiaron investigaciones y la segunda gran restructuración curricular que se consolidó a finales de 1992. Casi en la misma época se estableció el Regional Program of Biotechnology for Latin America and The Caribe - UNDP/UNESCO/UNIDO, que nos permitió ganar un proyecto bastante grande y equipar el laboratorio. En 1988 se inició también el Programa Andino de Biotecnología financiado por la Corporación Andina de Fomento (CAF) el cual no solo financió proyectos de investigación (la UNALM gano dos) sino que aceptó la propuesta de patrocinar y financiar inicialmente con 250 mil dólares un Programa Andino de Segunda Especialización en Biotecnología en la Facultad de Ciencias de la UNALM. El proyecto se elaboró con la participación de algunos profesores de biología, química, agronomía e industrias alimentarias: Existían los profesores, los fondos, se equiparían laboratorios, se especializaría a profesionales de Bolivia, Colombia, Ecuador, Perú y Venezuela y, en buena cuenta, hubiese fortalecido la carrera de biología y la generación de conocimientos. Sin embargo, en 1989, la propuesta no fue aprobada en el Consejo de Facultad y la CAF destinó los fondos a un instituto venezolano. Hay varias explicaciones a este error institucional que relataré y analizaré en el futuro pero una que se mencionó en ese momento es completamente inaceptable: algunos arguyeron que fue el temor a lo nuevo y desconocido, inaceptable, porque lo nuevo y lo desconocido son el motivo de la investigación y de la ciencia; el científico no teme a lo nuevo y a lo desconocido, lo enfrenta, lo clarifica, lo explica, abre camino.

\section{De 1993 al presente: El tercer currículo}

En la última fase de Proyecto de Asistencia al Desarrollo Institucional (PADI)/UNALM/USAID/Ministerio de Agricultura comprendida entre 1990 y 1992 se llevó a cabo la (segunda) gran reestructuración curricular de la UNALM. Después de un minucioso análisis sobre la formación de biólogos en el país que incluía la oferta y demanda de este tipo de profesionales, del grado de avance que se tenía en biología en la UNALM, en la suficiente diversidad profesional que aún tiene la Facultad de Ciencias, y pensando que la biología moderna también es, y ha sido, la base de carreras profesionales, desde la Comisión de Currículo que presidía sometí primeramente al Departamento de Biología el proyecto para el establecimiento de una nueva carrera profesional, adicional a la carrera de biología, denominada Ingeniería Biológica que no implicaba la creación de un nuevo departamento, ni la extinción de otro y, muchos menos, la cancelación de la carrera de Biología, que esperaba se reestructure acorde al desarrollo que había habido y a los que se avizoraba venían, así como tampoco hubo intención alguna de crear problemas. Por el contrario, usando términos biológicos, solo se trataba de un proceso de especiación.

Algunos consideran este momento como un "punto de quiebre” en la vida de la carrera (del departamento) de Biología y tal vez lo fue. En una crítica y dolorosa reunión de departamento para discutir la propuesta de la nueva Carrera de Ingeniería Biológica, ésta no fue aprobada. Las razones que pudieron haber conducido a rechazar esta nueva carrera las dejo para una versión posterior y más detallada de este relato. Es menester volver a mencionar, como en aquella ocasión lo hice, que la carrera de Ingeniería Biológica no se trataba de una invención pues se empieza a gestar en el mundo desde 1941, en plena Segunda Guerra Mundial, cuando en el Instituto Tecnológico de Massachusetts profesores de los departamentos de microbiología y ingeniería química trabajaron juntos debido a la imperiosa necesidad de producir penicilina a gran escala para evitar las infecciones bacterianas que producían la muerte de los soldados aliados heridos, y que eran la causa del mayor número de decesos. En esta tarea también participaron los investigadores del USDA Northern Regional Research Laboratory (NRRL). Ni los microbiólogos ni los ingenieros químicos por separado podían lograr un escalamiento de los cultivos del hongo y recién lo lograron cuando ambos se juntaron y en 1943 se logró la producción masiva de 
la penicilina por la firma Pfizer. Con los años la carrera de Ingeniería Biológica se diseminó en todo el mundo. La Ingeniería Química surge como una "especiación" de la química en el s. XIX y ambas han desarrollado muchísimo y muy estrechamente sin que signifique la extinción de la química.

Sin embargo, se planteó como salida de conciliación la reestructuración de la carrera de biología en dos orientaciones: Biotecnología (pues ésta es una especialidad y no una carrera como lo es la Ingeniería Biológica) y Ecología. Solo algunas modificaciones se realizaron al plan curricular de Ingeniería Biológica para compatibilizarlo con Ecología. A partir de entonces la Facultad de Ciencias a través de la Comisión de Currículo, organizó muchas reuniones facultativas para discutir los nuevos currículos tanto en el campus como fuera del mismo, participando también egresados. El nuevo y actual currículo de Biología fue aprobado y empezó a funcionar en 1993. Parece ser, y sobre esto no hay mucha certeza, que debido a que la Facultad de Ciencias había solicitado opinión externa acerca del nuevo currículo, un profesor de una universidad privada lo presentó como propio en un Congreso de Biología en México, siendo premiado como currículo modelo. Afortunadamente, aquella universidad nunca lo pudo implementar. El ejemplo de la UNALM ha servido para que la biotecnología sea considerada en los currículos de biología de varias universidades del país, inclusive como carreras separadas en algunas universidades.

En 1997, en su último ańo de estudios, la primera promoción de estudiantes del área de Biotecnología (ingresantes en 1993) solicitó la implementación de Ingeniería Biológica como carrera profesional y la posibilidad de titularse como Ingenieros Biólogos. Después de varias semanas de debates y discusiones, incluyendo la exposición ante el Consejo de Facultad del Prof. Andrés Illanes de la Universidad Católica de Valparaíso, Chile, y la opinión escrita de varios especialistas latinoamericanos, en sesión extraordinaria del Consejo de Facultad del 2 de diciembre de 1997, presidida por su Decano, el Biol. M.Sc. César Guerrero, se aprobó la creación de la Carrera de Ingeniería Biológica permitiendo, también, que los estudiantes de la primera promoción de Biotecnología que quieran acogerse al mismo podrían hacerlo cursando unos pocos cursos que serían nuevos en su plan de estudios. La Facultad de Ciencias emitió la resolución № 2855/97-FC-UNALM de fecha 3 de diciembre de 1997, la cual fue enviada al Consejo Universitario para su ratificación, cosa que nunca ocurrió aunque la resolución tampoco fue derogada. Fue el entonces Vicerrector Académico, Ing. Hugo Nava, quien evitó que el Consejo Universitario vea el caso. Una razón para esto, aparentemente, fue el interés de éste por crear la Carrera de Ingeniería Ambiental, lo cual realmente ocurrió. Es posible que haya habido otras fuerzas que también motivaron a incumplir el reglamento y no proceder con la ratificación de la resolución de la Facultad de Ciencias creando la Carrera de Ingeniería Biológica, lo cual será motivo de un análisis futuro también.

\section{El doctorado}

En el mundo ocurrían avances espectaculares tanto en la biología molecular como en la ingeniería biológica y en la biotecnología. La automatización de la técnica de Sanger para secuenciamiento del DNA dio inicio a la genómica, principalmente estructural, con el proyecto Genoma Humano en 1990, y ya en 1995 se pudo secuenciar el genoma de dos bacterias (Fleischmann 1995, Fraser 1995). Con la publicación de la técnica de Reacción en Cadena de la Polimerasa (PCR) en 1988, se abrió un campo de nuevos desarrollos no solo para la biología fundamental sino también para las aplicaciones en diversas áreas (Bartlett \& Stirling 2003). La clonación de la oveja Dolly en 1997 permitió entender algunos aspectos sobre la fisiología de células diferenciadas en lo fundamental y la posibilidad de establecer granjas génicas en lo aplicado (Wilmut et al. 1997). En 1996 se da inicio al cultivo comercial de plantas genéticamente modificadas que han sido adoptadas en varios países a tasas cercanas al 10\% anual (Marshall 2012). Varios compuestos químicos industriales empiezan a ser producidos biotecnológicamente y la ingeniería biológica empieza a enrumbarse hacia una incorporación de la genómica en el diseño y optimización de procesos (Chen 2012, Demain 2006, HattiKaul et al. 2007, Soetaert \& Vandamme 2006, Stottmeister et al. 2005). En el año 2001 se completa el secuenciamiento del genoma humano (IHGSC 2001). Hasta el 20 de octubre del 2012 se ha secuenciado los genomas de 3,707 especies - siendo el de la cebada el más reciente (IBSC 2012) - y otros 12,202 se encuentran en ejecución (http://www.genomesonline.org/ cgi-bin/GOLD/index.cgi). En la UNALM, que había avanzado mucho en procesos biotecnológicos, se adquiere en 1995 por medio de un proyecto financiado por el Programa Multinacional de Biotecnología de la OEA, un termociclador Perkin-Elmer para PCR, que fue, sino el primero, uno de los primeros en el Perú y se desarrollaron las primeras tesis sobre el tema, siendo una de las cuales la ejecutada con mi asesoría por la ahora Dra. Cecilia Sarmiento, Premio Nacional de Ciencias de Estonia.

En ese contexto, desde el año 2000, empieza a elaborarse un programa de doctorado que pretendía incorporar a la mayoría de doctores de la UNALM. Sin embargo, la gran mayoría de doctores de la UNALM no estuvieron dispuestos a colaborar con un programa de doctorado que sería básicamente de investigación. Esto dio lugar a que en el año 2002 se trabaje una propuesta basada en la Facultad de Ciencias y que se propuso como un Homenaje a la UNALM por el Centenario. Es necesario resaltar la invalorable colaboración de los profesores Inés Redolfi, Edgar Sánchez, Doris Zúñiga y David Campos (Fac. de Ind. Alimentarias) y de otros profesores. La propuesta planteó la creación del Programa Doctoral en Ciencias e Ingeniería Biológicas (PDCIB) conducente a la obtención del grado de Doctoris Philosophiae (Ph.D.) y dos especialidades iniciales: Ecología Aplicada e Ingeniería Biológica y Biotecnología (http://www. lamolina.edu.pe/doctorado/default.htm).

Las características del PDCIB son: a) que las vacantes se establecen de acuerdo a los proyectos financiados que tengan los profesores y que soporten la investigación doctoral; b) se requiere que los estudiantes se dediquen a tiempo completo; c) el primer semestre es común para todas las especialidades; d) para la disertación de la tesis es requisito haber publicado por lo menos un artículo científico en una revista ISI con factor de impacto mayor a 0,5 ; e) en contra de la corriente, los profesores del PDCIB no reciben remuneraciones económicas adicionales.

La propuesta fue rápidamente aprobada en el Departamento de Biología y en la Facultad de Ciencias. El primer escollo que hubo que vencer para la aprobación final fue en la Escuela de Postgrado, que luego de un constante ir y venir de observaciones y respuestas inmediatas, el proyecto fue aprobado. El segundo y más grande escollo fue a nivel del Consejo Universitario, 
cuyas autoridades principales eran opuestas a la idea de iniciar los estudios de doctorado en la UNALM. Finalmente, con la ayuda de algunos Decanos, se aprobó la creación del PDCIB el 31 de diciembre del 2002 con la respectiva resolución ( $\mathrm{N}^{\circ}$ 729-202-UNALM). La primera promoción ingresó en marzo del 2003 y la profesora del Departamento de Biología Gretty K. Villena fue la primera Ph.D. graduada en el PDCIB y en la UNALM. Es bueno resaltar que los egresados del PDCIB se gradúan con un promedio de 2.3 artículos en revistas ISI con un factor de impacto promedio de 2.137. Algunos de los egresados del PDCIB han ganado proyectos importantes financiados por INCAGRO, CONCYTEC y FINCyT.

\section{Algunos logros en biotecnología}

En los últimos 12 años los avances en ingeniería biológica y en biotecnología en el mundo han sido aún más espectaculares. La genómica funcional (transcriptómica, proteómica, metabolómica y flujómica) (Klamt \& Stelling 2003, Villena \& Gutiérrez-Correa 2008, Weld et al. 2006) se ha convertido en un tema fundamental que ha dado lugar a un conocimiento más integral sobre el funcionamiento de los seres vivientes y a una nueva visión de la biología, "Systems Biology" (Csete \& Doyle 2002, Ehrenberg et al. 2003, Kitano 2001, Nielsen \& Olsson 2002). De otro lado, este enfoque ha roto el mito que las células de cualquier organismo, unicelular o multicelular y complejo, se encuentran libres de estocasticidad (Acar et al. 2005); es todo lo contrario y los mecanismos moleculares de la resiliencia explican lo que comúnmente hemos definido como homeostasis. El concepto de resiliencia ha sido tomado de la ingeniería y ahora resulta muy útil en el nuevo enfoque que tiene la ingeniería biológica con resultados sorprendentes, aún en la ingeniería de ecosistemas (Berkenbusch \& Rowden 2003, Khun et al. 2010, Mee \& Wan 2012, Otero \& Nielsen 2010). Este enfoque se discute en detalle en el curso doctoral de Ingeniería Biológica.

La genómica funcional también ha dado lugar a una nueva visión de la ingeniería genética dándole un potencial más amplio convirtiéndose, más bien, en una "ingeniería genómica” (Carr \& Church 2009), pasando de la ingeniería metabólica (Graham et al. 2007, Lee et al. 2009, Stephanopoulos 2002, Ye \& Bathia 2012) y la evolución dirigida (Chatterjee \& Yuan 2006, Lin et al. 2009, Turner 2003) a la construcción de "factorías celulares" (Barnes \& Dickson 2006, Mapari et al. 2009, Pscheidt \& Glieder 2008, Villaverde 2010) y recientemente a la biología sintética (Aldrich et al. 2008, Endy 2008, Gibson et al. 2010, Sismour $\&$ Benner 2005). Otro desarrollo que vale la pena resaltar es el advenimiento de la metagenómica que está permitiendo por un lado, estudiar y entender más profundamente la ecología de microorganismos y, de otro, la identificación de nuevas especies microbianas hasta ahora elusivas a su estudio (Langer et al. 2006, Riesenfeld et al. 2004, Schmeisser et al. 2007, Warnecke \& Hugenholtz 2007). La "bioprospección molecular" permite la identificación de nuevos genes de interés económico a partir de diversos metagenomas (Grey et al. 2003, Handelsman 2005, Li et al. 2009, Lorenz \& Eck 2005).

Finalmente, los avances en biotecnología están cambiando el modelo económico que se aplicará en el futuro cercano: la bioeconomía, definida como "una economía basada en la biotecnología que usa materias primas renovables, particularmente biomasa y recursos genéticos, para producir productos y energía al menor costo ambiental" (Gutiérrez-Correa 2008, 2009).
Para continuar con este recuento es necesario hacer un paréntesis y remontarnos casi 25 años atrás. El 3 de junio de 1977, siendo aún Jefe de Práctica, formé el Laboratorio de Micología (desde 1987 Laboratorio de Micología y Biotecnología, LMB) con un grupo de estudiantes - Dr. Jorge Jhoncon, Dra. Doris Zúniga, MSc. Patricia Moreno, Biol. Norma Yague entre otros - para realizar investigaciones sobre ecología microbiana. Hasta 1987 publicamos cerca de 12 artículos cuyos temas son pioneros y con muy bajo seguimiento. En efecto, al escribir un artículo que acaba de ser publicado no pudimos encontrar nuevas investigaciones en el país de la magnitud de las que realizamos 35 años atrás ["Peru is a diverse country and has very broad microbial diversity richness yet little studied and exploited."] (Vega et al. 2012). En 35 años de actividad el LMB tiene más de 90 publicaciones. Hay que reconocer el aliento de profesores como P. Aguilar, C. Morán, E. Loza, L. Basto y el apoyo financiero de Carlos López Ocaña, entonces Director de Centro de Investigaciones de Zonas Áridas de la UNALM.

En los últimos 10 años, por lo menos en el ámbito microbiano y genómico, la biotecnología en la UNALM ha avanzado sustancialmente y ya no se encuentra rezagada a nivel Latinoamericano y tampoco a nivel global. La Dra. Doris Zúniga ha dado un salto significativo con sus investigaciones que se iniciaron en ecología microbiana y microbiología del suelo hasta 1996 cuando incorpora la biología molecular a sus estudios sobre bacterias fijadoras de nitrógeno y de bacterias promotoras del crecimiento de plantas y los enrumba hacia la biotecnología produciendo buenos inoculantes que son aceptados por los agricultores y ha generado sinergias con la empresa privada (Álvarez-Martínez et al. 2009, Calvo et al. 2010, Ormeńo-Orillo et al. 2006, Santillana et al. 2008, Velezmoro et al. 2012). Asimismo, el grupo liderado por la Dra. Zúniga ha descrito una nueva bacteria, Pseudomonas punonensis sp. nov., a partir de pajas de pastos donde se seca la papa en la preparación de "tunta" (Ramos et al. 2012). Recientemente, la Dra. Zúñiga está utilizando también herramientas de ingeniería biológica para el modelamiento y optimización de los cultivos de estas bacterias en biorreactores para aumentar la eficiencia y reducir los costos de producción de bioinoculantes. La Dra. Doris Zúniga y su grupo (Dra. Carmen Velzmoro - profesora del PDCIB - y la Biol. K. Ogata) han ganado varios proyectos tanto nacionales como internacionales (CONCYTEC, FINCyT, FIDECOM y otros) con los que ha re-equipado su laboratorio y puede hacer investigaciones de calidad convirtiéndose en un referente latinoamericano en esta área de la biotecnología agrícola, además de asesorar 3 tesis de doctorado del PDCIB. En mérito a sus investigaciones la Dra. Doris Zúníga recibió en el 2011 el "Premio L'Oreal por la Mujer en la Ciencia".

Por nuestra parte en el LMB, entre 1982 y 1987 tuvimos un periodo de transición desde la ecología microbiana hacia la biotecnología industrial. Hasta el año 2000, las investigaciones que realizamos estuvieron referidas al desarrollo de sistemas de producción de enzimas con cultivos mixtos y biorreactores de estado sólido, siendo pioneras en esta área y los artículos publicados siguen siendo citados con bastante frecuencia (Castillo et al. 1994, Dueñas et al. 1995, Gutiérrez-Correa 1999, Gutiérrez-Correa \& Tengerdy 1997, 1998, 1999, GutiérrezCorrea et al. 1999).

En los últimos 12 ańos en el LMB desarrollamos con la Dra. Gretty K. Villena un nuevo sistema de producción de enzimas 
más eficiente (biopelículas de hongos filamentosos) basado en el proceso de adhesión celular como una variante, conjuntamente con la fermentación en estado sólido, de una nueva categoría fermentativa que denominamos Fermentación por Adhesión a Superficies (Gutiérrez-Correa \& Villena 2003, 2010, Gamarra et al. 2010). Como consecuencia de una publicación nuestra en el 2006 (Villena \& Gutiérrez-Correa, 2006), otros grupos en el mundo utilizaron nuestra propuesta (y particularmente un grupo escoses inició colaboraciones con nosotros) para entender y desarrollar tratamientos a micetomas pulmonares producidos por $A$. fumigatus, que se demostró formaban también películas en los pulmones y eso los hacía muy virulentos. En el 2011 publicamos conjuntamente un compendio relacionado a los aspectos clínicos (Ramage et al. 2011) y en el 2012 otro relacionado a los aspectos básicos e industriales (Gutiérrez-Correa et al. 2012). Otros grupos en Brasil, Canadá, India y otros paises están usando nuestro sistema para desarrollar procesos de producción de varias enzimas.

El tema de la fermentación por adhesión a superficies y, en particular de la biopelículas de hongos filamentosos, nos ha llevado a desarrollar y profundizar en la genómica funcional, particularmente en transcriptómica (Villena et al. 2009a) y proteómica habiendo publicado el primer artículo en el mundo sobre el proteoma de biopelículas de Aspergillus niger (Villena et al. 2009b) y un análisis del transcriptoma global usando microarreglos de DNA se encuentra en preparación. La necesidad de optimizar el proceso de producción de enzimas por biopelículas de $A$. niger ha sido el aliciente para profundizar en estudios básicos moleculares de diferenciación celular, regulación de la expresión de genes - un nuevo sistema de regulación se encuentra en evaluación - y otros aspectos usando técnicas moleculares nuevas como RNAseq y RNAi.

Desde el año 2008 iniciamos investigaciones sobre bioprospección molecular por lo que hemos aislado, clonado y secuenciado un número de genes de importancia económica, algunos de los cuales servirán para la construcción de factorías celulares. Hemos generado vínculos sinérgicos con empresas y gracias a varios proyectos ganados últimamente (FINCyT, CONCYTEC, FIDECOM y contratos de investigación) se ha re-equipado el LMB con equipos de última generación para transcriptómica, proteómica y metabolómica. Aparte de un buen número de tesis de titulación y de tesis de maestría de programas nacionales e internacionales (USA y Holanda), se han desarrollado o se llevan a cabo 6 tesis de doctorado del PDCIB y hemos dado entrenamiento avanzado a estudiantes graduados de Holanda, Japón, Francia y Gabón. En mérito a sus investigaciones la Dra. Gretty K. Villena, actual Coordinadora del PDCIB, recibió este año 2012 el "Premio L'Oreal por la Mujer en la Ciencia". Conjuntamente con el grupo de la Dra. Zúñiga en los últimos 12 años hemos publicado 28 artículos científicos en revistas científicas ISI con factor de impacto.

Otros grupos de investigación del Departamento de Biología (Dra. Rosa Espejo - profesora del PDCIB - M.Sc. César López y M.Sc. Roberto Mansilla) y de la Facultad de Agronomía (Dr. Raúl Blas, profesor del PDCIB) en el área de plantas realizan investigaciones en marcación molecular de especies nativas (Blas et al. 2008, Blas \& Petrescu 2009, Mane et al. 2008, de la Torre et al. 2008). Igualmente, el grupo de la Facultad de Industrias Alimentarias conformado por el Dr. David Campos, la Dra.
Rosana Chirinos - ambos profesores del PDCIB - y otros profesores realizan investigaciones muy importantes sobre metabolitos de plantas nativas generando grandes oportunidades para la utilización económica de la biodiversidad vegetal (Chirinos et al. 2007, 2008, 2009, 2010).

El desarrollo de la biotecnología en la UNALM ha influenciado el desarrollo de esta área en el Perú no solo en las adaptaciones curriculares que se han producido en varias universidades del país sino también en las investigaciones biotecnológicas que se llevan a cabo en universidades e institutos de investigación en las diferentes regiones del país (Gutiérrez-Correa 2005, GutiérrezCorrea \& Estrada 2008).

\section{Conclusiones}

Aunque este relato ha sido realizado para recordar el camino que ha seguido la biotecnología en la UNALM, ha sido necesario remontarse casi al origen de la Carrera de Biología. En el transcurso de 50 años, se han formado biólogos con tres currículos diferentes y en todos los casos han demostrado una buena formación. Así como tenemos egresados de los dos primeros currículos que dejan muy en alto a su Alma Mater en investigación y en la actividad profesional en institutos, universidades y empresas del país y del extranjero (EEUU, Suecia, Suiza, Finlandia, Francia, Estonia y otros países), también los tenemos del último currículo, en este caso del área de biotecnología que es lo que yo conozco, que también están dejando muy en alto a la UNALM y que debido a la sólida formación en ciencias que han recibido no solo lo hacen muy bien en los temas más comunes de la biotecnología sino también en temas como medioambiente, cáncer y aún en neurobiología.

El país ha cambiado y el mundo ha cambiado. A diferencia de los años 80's y 90's en que solo había dinero para investigar fuera del país pero aún así alcanzable, ahora hay dinero en el país, no muchísimo por cierto, y llama la atención que la UNALM, particularmente la Facultad de Ciencias, tenga muy poca participación en los concursos de proyectos y muchos profesores sigan esperando que la universidad les proporcione fondos para investigar de un magro presupuesto que debe destinarse a la enseñanza práctica. Así mismo, preocupa que algunos biólogos se olviden de la biología cuando se enfrentan a problemas que son fácilmente explicados por ella o se vuelvan fundamentalistas como si la biología fuese una ideología dogmática.

Los profesores fundadores como el Dr. Pedro Aguilar y todos los de la primera generación, como fue dicho al inicio, son los que crearon el espíritu de la carrera y, es mi opinión, que son ellos los que deben recibir, con todo merecimiento, todos los homenajes por el Cincuentenario; sus alumnos y los nuevos solo hemos seguido sus enseñanzas y su espíritu, no siempre bien por cierto. Relatar la historia de algo, cuando se ha sido parte de ella, implica no solo verificar hechos sino también recordar y, puesto que recordar es volver a vivir, se vuelve a vivir todo, lo bueno y lo malo, lo bonito y lo feo, las alegrías y las penas. Al recordar el camino de la biotecnología en la UNALM mi conclusión es que el balance es muy positivo. Es cierto, como ya han mencionado algunos colegas, que algunas personas nos han sido adversas de alguna manera pero esto no tiene que ver con la edad de ellos pues los he encontrado entre mayores y también, tristemente, en algunos jóvenes. Lo que realmente cuenta es, parafraseando a Winston Churchill, hacer de cada dificultad 
una oportunidad y es nuestra obligación seguir haciendo camino porque el país requiere de nosotros. No hay tiempo, a pesar del disgusto de algunos, para detenerse mucho tiempo a pensar la corriente epistemológica que se debe seguir pues, al fin de cuentas, son solo corrientes y una será cambiada por otra y así sucesivamente y, al final, la mejor corriente epistemológica es la que nos lleva mediante la investigación a encontrar verdades usables en la solución de los problemas del país y, también por qué no, del planeta.

\section{Agradecimientos}

Este relato está dedicado con mi eterno agradecimiento a los siguientes profesores fundadores e iniciadores de la Carrera de Biología de la UNALM en su 50 Aniversario: Julio Gaudron, Pedro Aguilar, Jacques Schonberg, O. Velarde, F. Anavitarte, E. Loza, C. López, C. Morán, A. Grobman, U. Moreno, A. Vejarano, L. Vega, F. Scheuch, R. Vallenas, A. Manrique, D. Roa, M. Vegas, J. De Albertis, V. Manrique, M. Peña, G. Rodríguez, L. Delgado de la Flor, A. Zea, E. Machado, C. Neyra, O. Vílchez, M. MacGregor y L. Basto.

\section{Literatura citada}

Acar M., A. Becskei \& A. van Oudenaarden. 2005. Enhancement of cellular memory by reducing stochastic transitions. Nature 435: 228-232.

Aguilar P. 1986. Breves notas históricas sobre la Facultad de Ciencias de la UNALM. http://www.biologia50unalm. com/2012/06/un-aporte-del-dr.html.

Aldrich S., J. Newcomb \& R. Carlson. Scenarios for the future of synthetic biology. Ind. Biotechnol. 4: 39-49.

Álvarez-Martínez E.R., A. Valverde, M.H. Ramírez-Bahena, P. García-Fraile, C. Tejedor, P.F. Mateos, N. Santillana, D. Zúñiga, A. Peix \& E. Velázquez. 2009. The analysis of core and symbiotic genes of rhizobia nodulating Vicia from different continents reveals their common phylogenetic origin and suggests the distribution of Rhizobium leguminosarum strains together with Vicia seeds. Arch. Microbiol. 191: 659-668.

Bartlett J.M.S. \& D. Stirling. 2003. A Short History of the Polymerase Chain Reaction. Meth. Mol. Biol. 226: 3-6.

Barnes L.M. \& A.J. Dickson. 2006. Mammalian cell factories for efficient and stable protein expression. Curr. Opin. Biotechnol. 17: 381-386.

Berkenbusch K. \& A.A. Rowden. 2003. Ecosystem engineering -moving away from 'just-so' stories. New Zealand J. Ecol. 27: 67-73.

Blas R., M. Ghislain, M.R. Herrera \& J.-P. Baudoin. 2008. Genetic diversity analysis of wild Arracacia species according to morphological and molecular markers. Genet Resour Crop Evol 55:625-642.

Blas R.H.S. \& D.C. Petrescu. 2009. Potato production and its constraints in Peru. AAB Bioflux 1: 53-57.

Calvo P., E. Ormeño-Orrillo, E. Martínez-Romero \& D. Zúñiga. 2010. Characterization of Bacillus isolates of potato rhizosphere from Andean soils of Peru And their potential PGPR characteristics. Braz. J. Microbiol. 41: 899-906.

Carr P.A. \& G.M. Church. 2009. Genome engineering. Nat. Biotechnol. 27: 1151-1162.

Castillo, M.R., M. Gutiérrez-Correa, J.C. Linden \& R.P. Tengerdy. 1994. Mixed culture solid substrate fermentation for cellulolytic enzyme production. Biotechnol. Lett. 16: 967-972.

Chatterjee R. \& L. Yuan. 2006. Directed evolution of metabolic pathways. Trends Biotechnol. 24: 28-38.

Chen G.-Q. 2012. New challenges and opportunities for industrial biotechnology. Microb. Cell Fact. 11:111 doi:10.1186/1475-2859-11-111.
Chirinos R., H. Rogez, D. Campos, R. Pedreschi \& Y. Larondelle. 2007. Optimization of extraction conditions of antioxidant phenolic compounds from mashua (Tropaeolum tuberosum Ruíz \& Pavón) tubers. Sep. Pur. Technol. 55: 217-225.

Chirinos R., D. Campos, N. Costa, C. Arbizu, R. Pedreschi \& Y. Larondelle. 2008. Phenolic profiles of andean mashua (Tropaeolum tuberosum Ruíz \& Pavón) tubers: Identification by HPLC-DAD and evaluation of their antioxidant activity. Food Chem. 106: 1285-1298.

Chirinos R., I. Betalleluz-Pallardel, A. Huamán, C. Arbizu, R. Pedreschi \& D. Campos. 2009. HPLC-DAD characterisation of phenolic compounds from Andean oca (Oxalis tuberosa Mol.) tubers and their contribution to the antioxidant capacity. Food Chem. 113: 1243-1251.

Chirinos R., J. Galarza, I. Betalleluz-Pallardel, R. Pedreschi \& D. Campos. 2010. Antioxidant compounds and antioxidant capacity of Peruvian camu camu (Myrciaria dubia (H.B.K.) $\mathrm{McVaugh}$ ) fruit at different maturity stages. Food Chem. 120: 1019-1024.

Csete M.E. \& J.C. Doyle. 2002. Reverse Engineering of Biological Complexity. Science 295: 1664-1669.

De Block M., L. Herrera-Estrella,M. Van Montagu, J. Schell \& P. Zambryski. 1984. Expression of foreign genes in regenerated plants and in their progeny. EMBO J. 3: $1681-1689$.

de la Torre A., C. López, E. Yglesias \& J.P. Cornelius. 2008. Genetic (AFLP) diversity of nine Cedrela odorata populations in Madre de Dios, southern Peruvian Amazon. For. Ecol. Manage. 255: 334-339.

Demain A.L. 2006. From natural products discovery to commercialization: a success story. J. Ind. Microbiol. Biotechnol. 33: 486-495.

Dueñas, R., R.P. Tengerdy \& M. Gutiérrez-Correa. 1995. Cellulase production by mixed fungi in solid substrate fermentation of bagasse. World J. Microbiol. Biotechnol. 11: 333-337.

Ehrenberg M., J. Elf, E. Aurell, R. Sandberg \& J. Tegnér. 2003. Systems Biology Is Taking Off. Genome Res. 13:2377-2380.

Endy D. 2008. Synthetic biology: Can we make biology easy to engineer? Ind. Biotechnol. 4: 340-351.

Fári M.G. \& U.P. Kralovánszky. 2006. The founding father of biotechnology: Károly (Karl) Ereky. Int. J. Hort. Sci. 12: 9-12.

Fleischmann R.D., M.D. Adams, O. White, R.A. Clayton, E.F. Kirkness, A.R. Kerlavage, C.J. Bult, J.F. Tomb, B.A. Dougherty, J.M. Merrick, et al. 1995. Whole-genome random sequencing and assembly of Haemophilus influenzae Rd. Science 269: 496-512.

Franklin R. \& R.G. Gosling. 1953a. Molecular configuration in sodium thymonucleate. Nature 171: 740-741.

Franklin R. \& R.G. Gosling. 1953b. Evidence for 2-chain helix in crystalline structure of sodium deoxyribonucleate. Nature 172: $156-157$.

Fraser C.M., J.D. Gocayne, O. White, M.D. Adams, R.A. Clayton, et al. 1995. The minimal gene complement of Mycoplasma genitalium. Science 270: 397-403.

Gamarra, N.N., G.K. Villena \& M. Gutiérrez-Correa. 2010. Cellulase production by Aspergillus niger in biofilm, solid-state and submerged fermentations. Appl. Microbiol. Biotechnol. 87: 545-551.

Gibson D.G., J.I. Glass, C. Lartigue et al. 2010. Creation of a Bacterial Cell Controlled by a Chemically Synthesized Genome. Science 329: 52-56.

Graham I.A., T. Larson \& J.A. Napier. 2007. Rational metabolic engineering of transgenic plants for biosynthesis of omega-3 polyunsaturates. Curr. Opin. Biotechnol. 18:142-147.

Gray K.A., T.H. Richardson, D.E. Robertson, P.E. Swanson \& M.V. Subramanian. 2003. Soil-based gene discovery: A new technology to accelerate and broaden biocatalytic applications. Adv. Appl. Microbiol. 52: 1-27. 
Gros F., F. Jacob \& P. Royer. 1979. Sciencies de la vie et société: Rapport au President de la République. La Documentation Française, Paris, 288p.

Gutiérrez-Correa M. 1983. Biología: guía de estudio. UNALM, Lima, 246p.

Gutiérrez-Correa M. 1985. Principios de Biotecnología. UNALM, Lima, 293p.

Gutiérrez-Correa M. 1999. Toward the understanding of fungal mixed culture solid substrate fermentation for cellulolytic enzyme production. Ph.D. Thesis, Gifu University, Japan.

Gutiérrez-Correa, M. 2005. Ciencias Biológicas, Bioquímica, Biología Molecular y Biotecnología en el Perú. En "La Investigación Científica y Tecnológica en el Perú”, CONCYTEC, Volumen I, pp.264-343, BCR-CONCYTEC, Lima.

Gutiérrez-Correa, Marcel. 2008. Bioeconomía (Primera Parte). Rev. Agronegocios 2(3): $14-17$.

Gutiérrez-Correa, Marcel. 2009. Bioeconomía (Segunda Parte). Rev. Agronegocios 3(1): 16 - 19.

Gutiérrez-Correa, M. \& R.P. Tengerdy. 1997. Production of cellulase on sugar cane bagasse by fungal mixed culture solid substrate fermentation. Biotechnol. Lett. 19: 665-667.

Gutiérrez-Correa, M. \& R.P. Tengerdy. 1998. Xylanase production by fungal mixed culture solid substrate fermentation on sugar cane bagasse. Biotechnol. Lett. 20: 45-47.

Gutiérrez-Correa, M. \& R.P. Tengerdy. 1999. Cellulolytic enzyme production by fungal mixed solid substrate fermentation. Agro-food-Industry Hi-Tech 10: 6-8.

Gutiérrez-Correa, M., L. Portal, P. Moreno \& R.P. Tengerdy. 1999. Mixed culture solid substrate fermentation of Trichoderma reesei with Aspergillus niger on sugar cane bagasse. Bioresour. Technol. 68: 173-178.

Gutiérrez-Correa, M. \& G.K. Villena. 2003. Surface Adhesion Fermentation: A new fermentation category. Rev. Peru. Biol. 10: 113-124.

Gutiérrez-Correa, M. \& R. Estrada. 2008. Línea base para la implementación del programa nacional en biotecnología agraria y agroindustrial en el Perú. En "Líneas de Base para la Implementación de Programas Estratégicos" (H. Fano \& M. Torres, eds.), pp. 121-140, INCAGRO, Lima.

Gutiérrez-Correa, M. \& G.K. Villena. 2010. Characteristics and techniques of fermentation systems. In "Comprehensive Food Fermentation and Biotechnology". (A. Pandey, C.R. Soccol, C. Larroche, E. Gnansounou, P. Nigam-Singh, eds.). Volume I, p.183-227. Asiatech Publisher, Inc. New Delhi.

Gutiérrez-Correa, M., Y. Ludeña, G. Ramage \& G.K. Villena. 2012. Recent advances on filamentous fungal biofilms for industrial uses. Appl. Biochem. Biotechnol. 167: 1235-1253.

Handelsman J. 2005. Sorting out metagenomes. Nat. Biotechnol. 23: $38-39$

Hatti-Kaul R., U. Törnvall, L. Gustafsson \& P. Börjesson. 2007. Industrial biotechnology for the production of bio-based chemicals -a cradle-to-grave perspective. Trends Biotech. 25: 119-124.

Herrera-Estrella L., A. Depicker, M. van Montagu \& J.Schell. 1983. Expression of chimaeric genes transferred into plant cells using a Ti-plasmid-derived vector. Nature 303: 209-213.

Hershey A.D. \& Martha Chase. 1952. Independent functions of viral protein and nucleic acid in growth of bacteriophage. J. Gen. Physiol. 31: 39-56.

International Human Genome Sequencing Consortium (IHGSC). 2001. Initial sequencing and analysis of the human genome. Nature 409: 860-921.

Jacob F. \& J. Monod. 1961a. On the regulation of gene activity. Cold Spring Harb. Symp. Quant. Biol. 26: 193-211.

Kitano, H.(Ed.). 2001. Foundations of Systems Biology. The MIT Press, Massachussets, 297p.

Klamt S. \& J. Stelling. 2003. Two approaches for metabolic pathway analysis? Trends Biotech. 21: 64-69.
Kuhn D., L.M. Blank, A. Schmid \& B. B.uhler. 2010. Systems biotechnology - Rational whole-cell biocatalyst and bioprocess design. Eng. Life Sci. 10: 384-397.

Langer M., E.M. Gabor, K. Liebeton, G. Meurer, F. Niehaus, R. Schulze, J. Eck \& P. Lorenz. 2006. Metagenomics: An inexhaustible access to nature's diversity. Biotechnol. J. 1: 815-821.

Lee S.Y., H.U. Kim, J.H. Park, J.M. Park \& T.Y. Kim. Metabolic engineering of microorganisms: general strategies and drug production. Drug Discov. Today 14: 78-88.

Li L.-L., S.R. McCorkle, S. Monchy, S. Taghavi \& D. van der Lelie. 2009. Bioprospecting metagenomes: glycosyl hydrolases for converting biomass. Biotechnol. Biofuels 2: 10 doi:10.1186/1754-6834-2-10.

Lin L., X. Meng, P. Liu, Y. Hong, G. Wu, X. Huang, C. Li, J. Dong, L. Xiao \& Z. Liu. 2009. Improved catalytic efficiency of Endo- $\beta$-1,4-glucanase from Bacillus subtilis BME-15 by directed evolution. Appl. Microbiol. Biotechnol. 82: 671-679.

Lorenz P. \& J. Eck. Metagenomics and industrial applications. Nat. Rev. Microbiol. 3: 510-516

Mane S.P., C.V. Robinet, A. Ulanov, et al. 2008. Molecular and physiological adaptation to prolonged drought stress in the leaves of two Andean potato genotypes. Funct. Plant Biol. 35: 669-688.

Marshall A. 2012. Existing agbiotech traits continue global march. Nat. Biotech. 30: 207.

Mapari S.A.S., A.S. Meyer, U. Thrane \& J.C. Frisvad. 2009. Identification of potentially safe promising fungal cell factories for the production of polyketide natural food colorants using chemotaxonomic rationale. Microb. Cell Fact. 8: 24 doi: $10.1186 / 1475-2859-8-24$

Mee M.T. \& H.H. Wang. 2012. Engineering ecosystems and synthetic ecologies. Mol. BioSyst. 8: 2470-2483.

Nielsen J. \& L. Olsson. 2002. An expanded role for microbial physiology in metabolic engineering and functional genomics: moving towards systems biology. FEMS Yeast Res. 2: 175-181.

Jacob F. \& J. Monod. 1961b. Genetic regulatory mechanisms in the synthesis of proteins. J. Mol. Biol. 3:318-56.

Ormeño-Orrillo E., P. Vinuesa, D. Zúñiga-Dávila, E. MartínezRomero. 2006. Molecular diversity of native bradyrhizobia isolated from Lima bean (Phaseolus lunatus L.) in Peru. Syst. Appl. Microbiol. 29: 253-262.

Otero J.M. \& J. Nielsen. 2010. Industrial systems biology. Biotechnol. Bioeng. 105: 439-460.

Pscheidt B. \& A. Glieder. 2008. Yeast cell factories for fine chemical and API production. Microb. Cell Factories 7: 25 doi: 10.1186/1475-2859-7-25.

Ramage, G., R. Rajendran, M. Gutiérrez-Correa, B. Jones \& C. Williams. 2011. Aspergillus biofilms: clinical and industrial significance. FEMS Microbiol. Lett. 324: 89-97.

Ramos E., M.H. Ramírez-Bahena, A. Valverde, E. Velázquez, D. Zúñiga, C. Velezmoro \& A. Peix. Pseudomonas punonensis sp. nov., a novel species isolated from grasses in Puno region (Peru). Int. J. Syst. Evol. Microbiol. Published online ahead of print September 21, 2012, doi: 10.1099/ ijs.0.042119-0.

Riesenfeld C.S., P.D. Schloss \& J. Handelsman. 2004. Metagenomics: Genomic analysis of microbial communities. Annu. Rev. Genet. 38: 525-552.

Santillana N., M.H. Ramírez-Bahena, P. García-Fraile, E. Velásquez \& D. Zúñiga. 2008. Phylogenetic diversity based on rrs, atpD, recA genes and $16 \mathrm{~S}-23 \mathrm{~S}$ intergenic sequence analyses of rhizobial strains isolated from Vicia faba and Pisum sativum in Peru. Arch. Microbiol. 189: 239-247. 
Schmeisser C., H. Steele \&W.R. Streit. 2007. Metagenomics, biotechnology with non-culturable microbes. Appl. Microbiol. Biotechnol. 75: 955-962.

Sismour A.M. \& S.A. Benner. 2005. Synthetic biology. Expert Opin. Biol. Ther. 5:1409-1414.

Soetaert W. \& E. Vandamme. 2006. The impact of industrial biotechnology. Biotechnol. J. 1: 756-769.

Stephanopoulos G. 2002. Metabolic engineering by genome shuffling. Nat. Biotechnol. 20: 666-668.

Stottmeister U., A. Aurich, H. Wilde, J. Andersch, S. Schmidt \& D. Sicker. 2005. White biotechnology for green chemistry: fermentative 2- oxocarboxylic acids as novel building blocks for subsequent chemical syntheses. J. Ind. Microbiol. Biotechnol. 32: 651-664.

The International Barley Genome Sequencing Consortium (IBSC). 2012. A physical, genetic and functional sequence assembly of the barley genome. Nature doi:10.1038/nature11543.

Turner N.J. 2003. Directed evolution of enzymes for applied biocatalysis. Trends Biotechnol. 21: 474-478.

Vega K, G.K. Villena, V.H. Sarmiento, Y. Ludeña, N. Vera, \& M. Gutiérrez-Correa. 2012. Production of alkaline cellulase by fungi isolated from an undisturbed rain forest of Peru. Biotechnol. Res. Int. vol. 2012, Article ID 934325, 7 pages, doi:10.1155/2012/934325.

Velezmoro C., E. Ramos, C. García \& D. Zúñiga. 2012. Genotypic identification of Bacillus sp. isolated from canned white asparagus (Asparagus officinalis) during the production/ processing chain in northern Peru. Ann. Microbiol. 62: 1207-1217.

Villaverde A. 2010. Nanotechnology, bionanotechnology and microbial cell factories. Microb. Cell Fact. 9: 53 doi:10.1186/1475-2859-9-53.

Villena G.K. \& M. Gutiérrez-Correa. 2007. Morphological patterns of Aspergillus niger biofilms and pellets related to lignocellulolytic enzyme productivities. Lett. Appl. Microbiol., 45: 231-237.

Villena G.K. \& M. Gutiérrez-Correa. 2008. Genómica funcional de hongos filamentosos. Bios 1: 28 - 31 .
Villena, G.K., T. Fujikawa, S. Tsuyumu, \& M. Gutiérrez-Correa. 2009a. Differential gene expression of some lignocellulolytic enzymes in Aspergillus niger biofilms. Rev. Peru. Biol. 15: 97-102.

Villena, G.K., L. Venkatesh, A. Yamazaki, S. Tsuyumu \& M. Gutiérrez-Correa. 2009b. Initial intracellular proteome profile of Aspergillus niger biofilms. Rev. Peru. Biol. 16: 101-108.

Villena, G.K., T. Fujikawa, S. Tsuyumu \& M. Gutiérrez-Correa. 2010. Structural analysis of biofilms and pellets of Aspergillus niger by confocal scanning laser microscopy and cryo scanning electron microscopy. Bioresour. Technol. 101: 1920-1926.

Warnecke F. \& P. Hugenholtz. 2007. Building on basic metagenomics with complementary technologies. Genome Biol. 8: 231 doi:10.1186/gb-2007-8-12-231.

Watson J.D. \& F.H.C. Crick. 1953a. A structure for deoxyribose nucleic acid. Nature 171: 737-738.

Watson J.D. \& Crick F.H.C. 1953b. Genetical implications of the structure of deoxyribonucleic acid. Nature 171: 964-967.

Weld R.J., K.M. Plummer, M.A. Carpenter \& H.J. Ridgway. 2006. Approaches to functional genomics in filamentous fungi. Cell Res. 16: 31-44.

Whittaker R.H. 1969. New concepts of kingdoms of organisms. Science 163: 150-160.

Wilkins M.H.F., A.R. Stokes \& H.R. Wilson 1953. Molecular structure of deoxypentose nucleic acids. Nature 171: 738-740.

Wilmut I., A.E. Schnieke, J. McWhir, A.J. Kind, K.H. Campbell.1997. Viable offspring derived from fetal and adult mammalian cells. Nature 385: 810-3.

Woese C.R., W.E. Balch, L.J. Magrum, G.E. Fox \& R.S. Wolfe. 1977. An ancient divergence among the bacteria. J. Mol. Evol. 9: 305-311.

Woese C.R., O. Kandler \& M.L. Wheelis. 1990. Towards a Natural System of Organisms: Proposal for the domains Archaea, Bacteria, and Eucarya. Proc. Nat. Acad. Sci. USA 87: 4576-4579.

Ye V.M. \& S.K. Bhatia. 2012. Metabolic engineering for the production of clinically important molecules: Omega-3 fatty acids, artemisinin, and taxol. Biotechnol. J. 7, 20-33.

Zakaib, G.D. 2011. Out on a limb. Nature 476: 20-21. 
